\title{
Some Initial Results and Observations from a Series of Trials within the Ofcom TV White Spaces Pilot
}

\author{
Oliver Holland, Shuyu Ping, Nishanth Sastry, \\ Hong Xing, Suleyman Taskafa, Adnan Aijaz \\ King's College London \\ London, UK \\ \{oliver.holland, shuyu.ping, nishanth.sastry, hong.xing, \\ suleyman.taskafa, adnan.aijaz\}@kcl.ac.uk \\ Mohammad Reza Akhavan, Julie McCann \\ Imperial College London \\ London, UK \\ \{reza.akhavan, jamm\}@imperial.ac.uk \\ Raymond Knopp, Florian Kaltenberger, \\ Dominique Nussbaum \\ Eurecom \\ Sophia Antipolis, France \\ \{raymond.knopp, florian.kaltenberger, \\ dominique.nussbaum\}@eurecom.fr
}

Juhani Hallio, Mikko Jakobsson, Jani Auranen, Reijo

Ekman, Jarkko Paavola, Arto Kivinen

Turku University of Applied Sciences

Turku, Finland

\{juhani.hallio, mikko.jakobsson, jani.auranen, reijo.ekman,

jarkko.paavola, arto.kivinen\}@turkuamk.fi

Ha-Nguyen Tran, Kentaro Ishizu, Takeshi Matsumura, Kazuo Ibuka, Hiroshi Harada

National Institute of Information and Communications

Technology (NICT)

Yokosuka, Kanagawa, Japan

\{haguen, ishidu, matsumura, ibuka, harada\}@nict.go.jp

\author{
Pravir Chawdhry, Jean-Marc Chareau, James Bishop, \\ Michele Bavaro, Philippe Viaud, Tiziano Pinato, \\ Emanuele Anguili \\ Joint Research Centre of the European Commission \\ Ispra, Italy \\ \{pravir.chawdhry, jean-marc.chareau, james.bishop, \\ michele.bavaro, philippe.viaud, tiziano.pinato, \\ emanuele.anguili\}@jrc.ec.europa.eu \\ Yue Gao, Zhijin Qin, Qianyun Zhang \\ Queen Mary, University of London \\ London, UK \\ \{yue.gao, z.qin, qianyun.zhang\}@qmul.ac.uk \\ Rogerio Dionisio, Jose Ribeiro, Paulo Marques \\ Institute of Telecommunications \\ Aveiro, Portugal \\ \{rdionisio, jcarlosvgr, pmarques\}@av.it.pt \\ Heikki Kokkinen \\ Fairspectrum Oy \\ Helsinki, Finland \\ heikki.kokkinen@fairspectrum.com \\ Tomaž Šolc, Mihael Mohorčič \\ Jožef Stefan Institute \\ Ljubljana, Slovenia \\ \{tomaz.solc, miha.mohorcic\}@ijs.si \\ Keiichi Mizutani, Hiroshi Harada \\ Kyoto University \\ Kyoto, Japan \\ \{mizutani, hiroshi.harada\}@i.kyoto-u.ac.jp
}

\section{INTRODUCTION}

Research in TV White Spaces (TVWS) has accelerated rapidly ever since the FCC produced its initial opinion on rules for White Space Devices (WSDs) in November 2008. After much regulatory tweaking [1], [2], and initial deployments of such devices in the US, Europe is following with the finalization of rules and testing of TVWS technology on a large scale [3]-[6]. This is particularly driven by the UK regulator Ofcom's work and instantiation of a large pilot of WSDs and the underlying enabling technology [6]. All trials within this pilot must operate under Ofcom's prospective requirements for WSDs, reflected in ETSI 301598 [5].

Operating under the UK/EU rules [5], the Ofcom Pilot serves a number of purposes and objectives, such as:

- Provision a proof of concept of the TVWS framework.

- Verification before commercial TVWS operations start. 
- Involvement of the regulator, industry, and end users in the process, such that the interactions between the relevant stakeholders can be verified.

The Ofcom Pilot also aims to test several aspects, such as:

- Device operations.

- Geolocation Database (GDB) contract qualification.

- GDB operation and calculations.

- Ofcom's provision of the qualifying GDB listing.

- Ofcom's DTT calculation results and provision of Programme Making and Special Events (PMSE) data.

- Interference management.

- Coexistence.

In practice, this further includes verification of other aspects, such as the testing methodology for WSD RF performances, testing methodology for WSDs interactions with Ofcom's "database of GDBs" and selection of the appropriate GDB to use, testing methodology for WSD interactions with the GDB (including aspects such as security), the testing methodology for correct operation of WSDs (e.g., RF channel/power settings based on information from the GDB, ceasing to transmit when communication with the Ofcom "database of GDBs" or the GDB itself is not successfully carried out, changing of RF channels and powers if necessary, based on changed information from the GDB, etc.), the methodology for monitoring interference and the correctness of interference levels around deployments of WSDs under the Ofcom Pilot, the assessment of any possible effects on primary services, and verification of security precautions, among other aspects. The correct performance of all of these elements is essential to the assurance of the viability of the wider picture of TVWS technology, and the confidence that the regulator is able to authorize such a technology for commercial use.

Our trials within the Ofcom Pilot are the subject of this paper, noting that reference [7] provides more information on our objectives. Some of our conformance testing work is described in Section II of this paper. Our range of WSDs and the locations being investigated are described in Section III. Section IV discusses deployment and performance testing scenarios, and research topics we are investigating. Section V presents some early results, before Section VI concludes.

\section{CONFORMANCE TESTING}

We expect that Ofcom is most interested in testing the validity of the underlying TVWS technology (e.g., the GDBs and interactions thereof) and the conformance of WSDs with certification requirements (i.e., compliance with ETSI 301598 [5]). This serves the key interest of the regulator in ensuring that the spectrum of primary services is adequately protected.

It is a requirement for all triallists participating in the Ofcom TVWS Pilot to certify their devices are performing according to ETSI 301 598, both in terms of RF aspects and in terms of logical aspects such as communication with the GDB and appropriate setting of parameters in accordance with responses from the GDB. Reaching beyond such requirements, our trials have very strong such testing capabilities and are undertaking a range of work on such conformance testing.

A wide range of equipment is available for conformance testing as a part of our trials. Some of the equipment, in particular the Rohde and Schwarz FSV series of spectrum analysers, for example, available at King's College London and at the Joint Research Centre of the European Commission, is able to perform measurements on Adjacent Channel Leakage Ratios (ACLR) directly, as configured by the user. This is a useful option to confirm performance in terms of the spectrum mask ("Class" of device, as specified by Ofcom/ETSI) and compare with the ETSI 301598 specified procedure, noting that ETSI 301598 requires that the spectrum analyser should merely be set to sweep the spectrum and output the observed values at a resolution bandwidth (RBW-i.e., in "chunks") of $10 \mathrm{kHz}$, which must then be post-processed to assess the RF performance of the WSD using a more complicated procedure. Given this, for the purpose of conformance assessment of WSDs, the key parameter of interest is typically the dynamic range of the spectrum analyser (aside from other more obvious parameters, such as accuracy and distortion performance, sensitivity and ability to set the RBW correctly to $10 \mathrm{kHz}$, among others). Further, it is noted that the ACLR measurements for performance Classes 1 and 3 are very challenging, typically requiring a high dynamic range spectrum analyser.

In terms of RF performance, Ofcom/ETSI specify 5 performance classes (see p. 15 of [5]). These performance classes compare power in the intended channel of width 8 $\mathrm{MHz}$ with power outside of the intended channel in $100 \mathrm{kHz}$ "chunks", and specify requirements in terms of the intended channel emissions $\pm 1, \pm 2$, and \pm 3 channels, with limits further out from \pm 3 channels being equal to those for the \pm 3 channel. Ofcom also specifies stringent requirements for assessment of emissions outside of the TV spectrum, for the range from 30 $\mathrm{MHz}$ up to $4 \mathrm{GHz}$. Importantly, these are the same for all WSDs, not dependent on class. Our trials have assessed such emissions and found devices to be compliant, however, there can be conformance issues close to the edges of the TV bands if the WSDs are transmitting on channels closest to those edges. Practically, Ofcom are not allowing WSDs to transmit in channels 21 and 60, those at the edges of the TV spectrum.

\section{TV White SPACE DEVICES AND DEPLOYMENT LOCATIONS}

Our trials have amassed a wide range of devices for use at various times:

- Three different forms of WSDs created by collaborators at NICT, Japan, namely: IEEE 802.11 af high-power and lowpower variant WSDs [8], and FD/TD-LTE base station and terminal WSDs [9]. These devices obtained white spaces information from NICT's geolocation database, which is included in the list of qualified databases by Ofcom. The database-device interface is compliant with PAWS.

- WSDs that are based on Eurecom ExpressMIMO2 software radios [10], driven by OpenAirInterface LTEMBMS waveforms (and perhaps, at a later stage, TD-LTE, 802.11af, and other waveforms) [11].

- Carlson RuralConnect devices [12], which use a proprietary waveform.

- InterDigital WiFi in TVWS devices, capable of aggregating up to 4 non-contiguous TV channels [13].

- KTS/Sinecom Agility White Space Radio [14] WSDs, which use a proprietary waveform. 
It is important to verify performance for a range of locations. Given our trials being driven by academics and research institutes, a large number of University campuses are made available for usage as part of the trials. These include:

- Numerous locations at King's College London campuses, including the Strand, Waterloo, Guys (London Bridge), St. Thomas (opposite Westminster), Denmark Hill, and Hampstead Campuses.

- Queen Mary University of London (Mile End, East London).

- University of York.

- University of Surrey (Guildford).

- Strathclyde University (Glasgow).

- Cambridge University.

- University of Bath.

These locations range from some of the most challenging that it is possible to envisage for operation of WSDs in the UK, such as at the Strand, close to perhaps the most extensive licensed PMSE usage in the world through West-End theatres, concert halls, television studios, etc., to less busy cases such as at the University of York, with a large, mainly rural, low population-density area to the South-East of the campus. Rooftop sites at locations including King's College London Denmark Hill, Queen Mary University of London, and others such as the University of York, allow for the investigation of relatively large-area provisioning in TVWS, and the option of point-to-point links, e.g., to provide backhaul via TVWS.

In addition to the wide-area coverage and point-to-point scenarios involving rooftop transmissions or installations, it is noted that numerous other likely scenarios for WSD deployment are covered by our trials and the range of locations available, including indoor coverage and indoor-tooutdoor coverage with a range of building characteristics, and of course a range of building characteristics and geometries with which to study outdoor-to-indoor coverage provision.

\section{DEPLOYMENT AND PERFORMANCE TESTING SCENARIOS, AND RESEARCH TOPICS}

Our trials are investigating a large number of deployment and performance testing scenarios, attempting to both play to the strengths of the wide range of WSDs that we have available, and to test a diversity of challenging cases for WSDs deployment. The following scenarios are anticipated:

- LTE Multicast/broadcast (eMBMS), using the Eurecom ExpressMIMO2/OpenAirInterface SDR equipment/software, and TVWS extensions to that developed with King's College London. A range of transmission coverage scenarios are investigated, from wide-area rooftop to a relatively limited area (indoors or ground level), dependent on the deployment locations and associated characteristics.

- TD-LTE in TVWS, using NICT LTE WSDs. Moderate coverage ranges are anticipated to be investigated.

- Broadband for Public Protection and Disaster Relief (PPDR) using Carlson Wireless WSDs.

○ Point-to-point links in TVWS, as might provide emergency backhaul in PPDR scenarios.

o Video surveillance.

- WiFi in TVWS (802.11af draft).

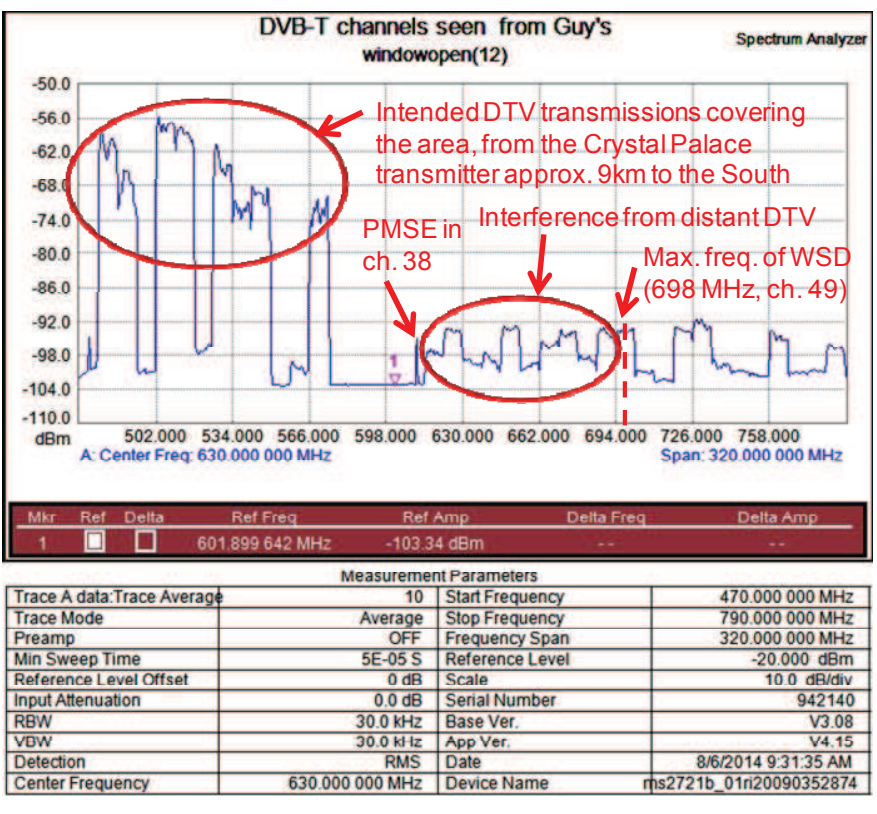

Fig. 1. A spectrum survey performed looking South from the King's College London Guys Campus hospital tower, clearly showing the intended TV transmissions covering the area, interference from distant DTV transmissions that are not meant to be covering the area, and other characteristics such as a PMSE device transmitting on the shared PMSE channel 38.

- Conventional wireless local-area coverage using low-power WiFi.

○ High-power WiFi for direct point-to-point links, again serving PPDR among other scenarios.

- M2M implementations.

- General broadband provisioning.

Being driven by academics and research institutes, a very strong emphasis is put on the research elements of our trials. The research studies that are being undertaken include:

- Solutions for Aggregation of resources/links (TVWS with licensed and unlicensed ISM, and within TVWS).

○ Qualitative and quantitative performance surveys.

- Secondary coexistence (e.g., LTE with 802.11af in TVWS).

- To undertake studies and surveys on the performances achieved, e.g., in terms of interference to primary TV services and PMSE services, and secondary performance through objective user opinion polling.

\section{SOME INITIAL RESULTS AND OBSERVATIONS}

Our trials have been running, in various phases of work, from July 2014. A number of observations can be reported.

\section{A. Scenarios for TV White Space Usage}

One initial observation, referring to Figure 1, has been that the busy nature of TV bands usage in London points to some particular applications being most useful/viable for TVWS. For scenarios where the WSDs are placed high above rooftops, a high degree of interference has been experienced towards WSDs, originating, for example, from distant primary (e.g., DTV) transmitters that are not meant to be covering the area. This is the case even for the many channels/locations at which WSDs are allowed to operate with maximum EIRP 
according to the Ofcom/ETSI framework. Given knowledge about the spatial TV channel usage mapping applied across the UK, it is anticipated that a similar situation exists across much of the UK, and particularly in areas where there is an overlap, or at the boundary, of TV broadcast station coverage areas.

This has implications for the viability of TVWS scenarios where WSD receivers are placed high above rooftops aiming to receive a low-power signal. For example, our $7 \mathrm{~km}$ pointto-point backhaul link between King's College London Denmark Hill and Queen Mary University of London has been affected significantly by this issue, with the interference from distant primary DTV stations (even in the many channels that the WSDs are maximum EIRP on) effectively reducing the received SINR from a viable/useable value (of typically slightly less than $10 \mathrm{~dB}$ ) by an order of magnitude to negative $\mathrm{dB}$ values or lower. Consequently, it is highly important to scan the spectrum for the best channel to use based on the interference situation, before choosing a channel, noting that some WSDs already support that capability. Indeed, our trial has observed that for this long-distance backhaul link case, it is far better to use an alternative channel that is allowed lower than maximum EIRP (in this case, TV channel 37, allowed 31 $\mathrm{dBm}-5 \mathrm{~dB}$ lower than the maximum EIRP according to the framework) than TV channels that are allowed a maximum EIRP of $36 \mathrm{dBm}$ (e.g., channel 48) in the GDB response.

We infer, based on such observations, that TVWS is perhaps most interesting in below roof-top cases, or cases where the propagation characteristics at TV frequencies can be used to greatly improve coverage in challenging cases, such as inside buildings and metro systems, among others.

\section{B. WSD Parameter Values and Parameter Acquisition}

Another key observation of our trials relates to the procedures for WSDs obtaining parameters, and the values of those parameters that are obtained. The Ofcom/ETSI framework specifies the concepts of master and slave devices, and specific and generic WSD operational parameters. The slave devices must obtain parameters via a master device, first forwarding their characteristics to the master device such that the master device can query the database on their behalf. The master device must transmit initial allowed parameters that any slave device can use anywhere within the coverage area of the master, such that the slave is able to transmit its characteristics to the master over those parameters. Parameters that allow this initial, "inspecific" transmission by slave devices are termed "generic" slave parameters, and parameters that are based on the later-obtained precise information from slave devices are termed "specific" slave parameters. An issue is that, given that generic slave parameters are effectively the worst case allowed power for any possible location within the master coverage area, their allowed powers are typically extremely low-so low as to not be usable even for the purpose of initial link formation. For example, in the challenging case of King's Strand Campus, for a master WSD transmitting at $31 \mathrm{dBm}$, the generic slave EIRP is lower than 3 $\mathrm{dBm}$ in all channels. This EIRP is not sufficient for the slave to transmit information to the master and the link be formed.

\section{WSD Performance Assessments}

First assessed are the long-distance links between King's College London Denmark Hill and Queen Mary University of

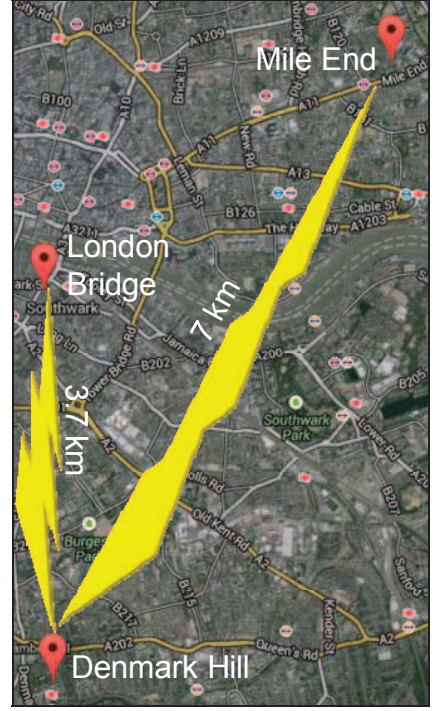

Fig. 2. The $7 \mathrm{~km}$ and $3.7 \mathrm{~km}$ long-distance links across London that have thus-far been tested in our trials.

London at Mile End (7 km distance), and King's College London Denmark Hill and King's College London Guys at London Bridge (3.7 km distance). These links are depicted in Figure 2. In both cases, channel 37 was used, for which the maximum allowed EIRP returned from the GDB was $31 \mathrm{dBm}$. This choice was because of aforementioned issues concerning interference to the WSDs from DTV, even on channels on which the absolute maximum EIRP of $36 \mathrm{dBm}$ was allowed. It is noted that the former $7 \mathrm{~km}$ link was only just able to be formed. Although there is optimisation that could be done on that link, the best rate that could be achieved was around 60 kbps over $7 \mathrm{~km}$, and the least challenging modulation and coding (BPSK with $1 / 2$-rate convolutional coding) could only achieve a BER of around 1-2\%. The best-case SINRs achieved were in the range of $8-10 \mathrm{~dB}$. The $3.7 \mathrm{~km}$ link enjoyed far better performance, where 16-QAM 1/2-rate convolutional coding achieved a BER of $10^{-6}$. Lab testing implies this leads to a downlink rate of $6.4 \mathrm{Mbps}$, and uplink rate of 5.1 Mbps.

Another area of performance assessment has been for indoor broadband provisioning, e.g., providing indoor pointto-point backhaul for broadband access points. This assessment has been done at the Strand Campus of King's College London, which is valuable for such as effort given its wide range of building types and implementable scenarios. Figure 3 depicts the layout of the parts of the Strand and King's buildings in the Strand Campus. Four links have been tested. Link 1 is from the "Flexible Radio" lab of the Centre for Telecommunications Research at King's College London to the first author's office, on the same floor and through some 4-5 walls including a closed metal blind covering a high-loss glass wall at the author's office. The distance of the direct path for Link 1 is approximately $10 \mathrm{~m}$. Link 2 is from the lab to the "Old Committee Room" in the King's Building, some $20 \mathrm{~m}$ away over a partial change in floor level, noting that the King's Building is of very rugged stone construction. Link 3 is across numerous rooms/walls to the "Refectory", some $80 \mathrm{~m}$ away on the same level of the King's building as the Old Committee room. Link 4 is to a classroom on the second floor 
of the Strand Building, transmitting diagonally up through at least 3 walls/floors, and across by some $10 \mathrm{~m}$.

Initial results are in terms of the performance for various modulation and coding rates, using the Carlson RuralConnect WSDs. It is noted that the Carlson devices are capable of 16QAM, QPSK and BPSK modulation, and convolutional coding rates of $1 / 2$ and $3 / 4$, or indeed transmission with no coding applied. Before any tests were done, a first assessment was the achievable performance for a (near-)ideal link, through transmission in the same room between the base station and terminal, with antennas directed away from each other and the transmission power attenuated by $19 \mathrm{~dB}$ such as to ensure that the receive radio was not saturated/compressed by the high signal level. The SINR observed by the receive radio in this case was $34.8 \mathrm{~dB}$. We assessed this link for a number of minutes, using the highest rate modulation (16QAM) and no coding. In the entire duration that the link was assessed, not a single bit (hence frame) error occurred.

First testing Link 1, the performance for 16-QAM with no coding and with $1 / 2$ coding rate, is shown in Figure 4 . No other modes were tested as it was found that the WSDs were able to achieve sufficient performance in the most-challenging modes of operation hence would be even better in other less challenging modes. Moreover, for Link 1, the WSDs anyway default to 16-QAM with no coding applied if configured to automatically select modulation and coding scheme. These results were obtained by extracting statistics from the device on a per-second basis, and the resulting values used to form the CDFs in Figure 4. Moreover, it is noted that the radios of the devices operated at an output of $20 \mathrm{dBm}$, the feeder cable loss was $1 \mathrm{~dB}$, and the antenna gain was $11 \mathrm{~dB}$. This gave an EIRP from the setup of $30 \mathrm{dBm}$. The devices were set to use TV channel 37, noting that only channels 27 and 37 were viable for Carlson Class 3 WSD usage at the Strand. For the location/height at the Strand that the work was done, the GDB allowed a maximum power of $31 \mathrm{dBm}$ for both these channels.

Referring to the 16-QAM with no coding case, the average BER from the distribution presented in Figure $4(\mathrm{a}, \mathrm{b})$ was $2.7 * 10^{-3}$, noting that the average SINR that the receiver saw in this case was $29.7 \mathrm{~dB}$. The average frame success probability was $95 \%$. With $1 / 2$-rate convolutional coding applied (Figure $4(\mathrm{c}, \mathrm{d}))$, the average BER was reduced to $1.2 * 10^{-3}$ despite the average SINR seen by the receiver for this experiment being reduced to $28.8 \mathrm{~dB}$. The average frame success probability was increased to $99 \%$. It is inferred that the reduction in average SINR seen for this latter case was caused by activity in the building, e.g., people moving and obstructing paths between the transmitter and receiver. Moreover, the rate that the Link achieved, with the devices operating in automatic modulation and coding selection mode and the link being stress-tested using a number of speed testing tools, was in the range of $6.5-8.3 \mathrm{Mbps}$ in the downlink, and 2.6-3.2 Mbps in the uplink. It is noted that a radio-firmware update has been made available for the Carlson devices, which has been applied and the rates tested again until this. The firmware update improved the downlink rate to somewhere in the range of 10.0-11.5 Mbps; the uplink rate was unchanged.

Moving on to Links 2-4, Link 2 achieved a performance of in the range of 5.7-9.9 Mbps on the downlink, and 1.0-2.2 Mbps on the uplink. It is noted that the high range of achieved

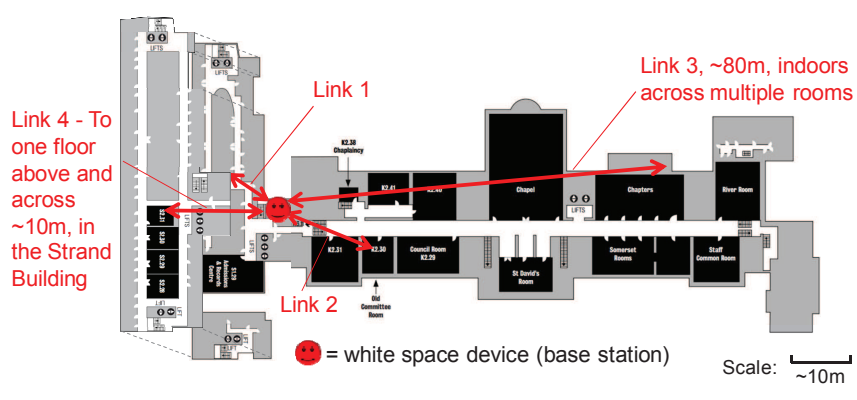

Fig. 3. Indoor room plans of the Strand and King's buildings (combined) of the King's College London Strand Campus. The Strand Building is to the left of the WSD, and the King's Building is to the right. Both the $1^{\text {st }}$ and $2^{\text {nd }}$ floors of the Strand Building are depicted, whereas only the $2^{\text {nd }}$ floor of the King's Building is depicted.

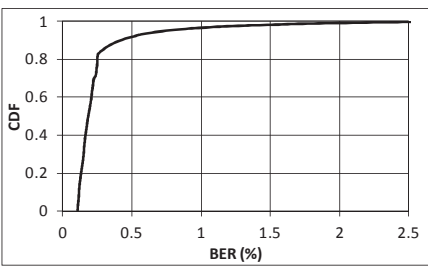

(a)

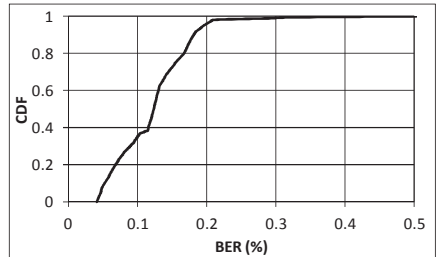

(c)

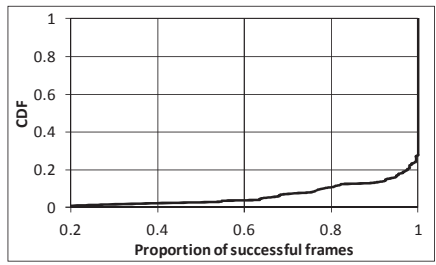

(b)

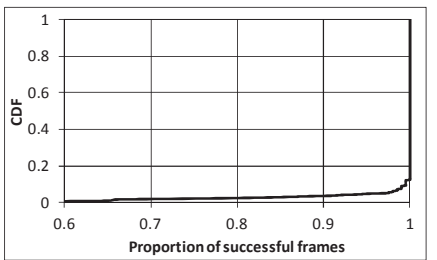

(d)
Fig. 4. Modulation and coding testing results for Link 1: (a) BER for 16QAM with no coding, (b) frame success probability for 16-QAM with no coding, (c) BER for 16-QAM with 1/2-rate convolutional coding, (d) frame success probability for $16-\mathrm{QAM}$ with $1 / 2$-rate convolutional coding.

rates was due to the link falling back to 16-QAM with $1 / 2$-rate convolutional coding both on the downlink and uplink during the testing. Interestingly, in coding/modulation testing 16QAM with no coding achieved a bit error rate of $1.1 * 10^{-8}$, and an average frame success probability of $99.98 \%$ (to two d.p.). This is despite the average observed SINR at the receiver being only $26.6 \mathrm{~dB}$. For reasons of such good performance with the most challenging modulation and coding scheme, further testing of modulation and coding schemes that were less challenging for this link was not done. Moreover, two observations are proposed related to the observed performance for this link. First, the high variability in performance was due to activity in the building hence attenuation by students and staff, noting that the initial modulation/coding link testing that demonstrated excellent performance was done in August when the building was almost empty, whereas the later link rate stress-testing was done in October when the building was extremely busy and there was a high variability of students and staff using the corridors/rooms (this ranged from the corridors/rooms being almost empty, to being extremely busy, often changing within the timescale of a few minutes). Second, it seems likely that Link 2 had time-diversity 
characteristics that were extremely favourable for the Carlson WSDs, compared with, say, Link 1, leading to the exceptional performance of Link 2 in the best-case experiments, despite the reduced observed SINR compared with Link 1.

Regarding Link 3, the performance of this link was extremely variable depending on the optimal placing and orientation of the antennas at each end of the link, noting that we only used orientations where the antenna was pointed directly towards the receive radio, or varied somewhat by a maximum of $90^{\circ}$ to that direction. For example, with BPSK modulation and no coding, by optimising the antenna position/origination at each end of the link the bit error rate could be reduced from approximately $5 * 10^{-2}$ to approximately $2 * 10^{-6}$, more than a reduction of a factor of 10,000 . It is noted that through varying antenna positions on this link, it was possible to achieve good performance even with 16-QAM modulation and no coding. This is reflected in the rates that were achieved under testing of the devices, in the range of 1.19.8 Mbps on the downlink, and 0.1-1.2 Mbps on the uplink.

Finally, Link 4 was able to achieve a near-perfect performance. The observed SINR on the downlink was 29.4 $\mathrm{dB}$, and the observed SINR on the uplink was $31.2 \mathrm{~dB}$. Noting that testing was done using the new firmware for the devices, the achieved downlink rate was in the range 10.9-11.6 Mbps. The uplink achieved rate was in the range 1.7-2.3 Mbps.

\section{White Space Capacity and Aggregation Studies}

Key questions for TVWS are: How much white space is there? And what can be achieved using that white space? These are all the more important to answer for the UK case, which operates under significantly different rules from the US.

To shed some light on this, we have investigated the available white space in the London, UK area, and also the optimum capacity that can be achieved by aggregating all of that white space. Our studies have sampled white space availability according to the UK framework in a rectangular grid defined by the top-left corner (latitude, longitude) 51.678064, -0.506744, and the bottom-right corner 51.312133, 0.22934 , with a sampling frequency of $0.01 \square$ both in latitude and longitude. This equates to the area approximately as bounded by the London M25 orbital motorway/highway, and 2,775 sampled locations within that area.

TABLE I: NUMBER OF CHANNELS AVAILABLE IN THE LONDON M25 AREA WITH ALLOWED POWER $>=30 \mathrm{dBm}$ EIRP, FOR A TRANSMITTER HEIGHT $30 \mathrm{~m}$.

Number of channels

\begin{tabular}{|l|c|c|c|c|c|}
\cline { 2 - 6 } \multicolumn{1}{c|}{} & Class 1 & Class 2 & Class 3 & Class 4 & Class 5 \\
\hline Average & 15.6 & 15.4 & 15.2 & 12.6 & 10.2 \\
\hline STD & 8.4 & 8.4 & 8.5 & 8.1 & 7.1 \\
\hline CoV & 0.54 & 0.55 & 0.56 & 0.64 & 0.70 \\
\hline
\end{tabular}

TABLE II: RATE ACHIEVED AMONG LONDON M25 AREA LOCATIONS AGGREGATING ALL AVAILABLE WHITE SPACE AT MAXIMUM ALLOWED POWER IN EACH CHANNEL. 2 km LINK, TRANSMITTER HEIGHT $30 \mathrm{~m}$, RECEIVER HEIGHT $1.5 \mathrm{~m}$, SHANNON EFFICIENCY 0.5, HATA URBAN PATH LOSS.

Achieved Rate (Mbps)

\begin{tabular}{|l|c|c|c|c|c|}
\cline { 2 - 6 } \multicolumn{1}{c|}{} & Class 1 & Class 2 & Class 3 & Class 4 & Class 5 \\
\hline Average & 167.0 & 165.1 & 155.4 & 130.9 & 104.7 \\
\hline STD & 84.2 & 84.4 & 82.5 & 77.4 & 66.8 \\
\hline CoV & 0.50 & 0.51 & 0.53 & 0.59 & 0.64 \\
\hline
\end{tabular}

Using this methodology, Table 1 gives the average number of channels that are available in the M25 area, for all 5 spectrum mask classes, as well as the standard deviation and coefficient of variation in the number of channels. This is for a transmitter at $30 \mathrm{~m}$ height above ground level, with an allowed power of $30 \mathrm{dBm}$ EIRP or more. Table 2 gives the equivalent average rates (again including the standard deviation and coefficient of variation) that can be achieved over a $2 \mathrm{~km}$ link, aggregating all available channels at maximum allowed power, with the receiver at a height of $1.5 \mathrm{~m}$ and Shannon efficiency of 0.5 , using the Hata urban path loss model. This configuration pertains to a local-area mobile broadband provision scenario through TVWS.

The results in Tables 1 and 2 show an average of 10-15 TV channels being available, and average aggregate rates of around 100-170 Mbps. Moreover, it is noted that Class 1-3 devices will perform relatively similarly, whereas Class 4 and 5 devices will perform worse both in terms of average number of channels/rates, and uncertainty in number of channels/rates.

\section{E. Coexistence with Primary Services}

Some initial experiments assessing coexistence with both DTV and PMSE devices have been done. For the DTV coexistence experiments, two antenna configurations have been investigated with the WSD antenna and the DTV receive antenna mounted on the same pole, less than $10 \mathrm{~cm}$ apart. All of this work has been done at King's College London Denmark Hill campus. In one case, the WSD antenna and the PMSE receiver were pointed in different directions, and in the other case they were pointed in the same direction in order to increase coupling between them. In all assessed cases, the WSD was operating at maximum allowed power in the adjacent channel to the DTV signal that was received. The DTV signal was being received direct line-of-sight from the Crystal Palace TV transmitter which was located $5 \mathrm{~km}$ away to the South. Moreover, the Crystal Palace TV transmitter was transmitting with a power of $200 \mathrm{~kW}$ ERP in each of the TV channels that we assessed. Figure 5 depicts our antenna configurations for this work.

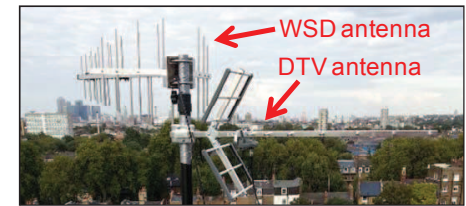

(a)

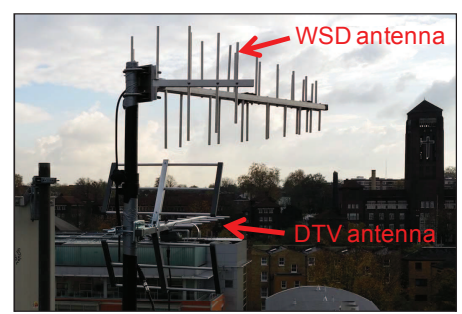

(b)

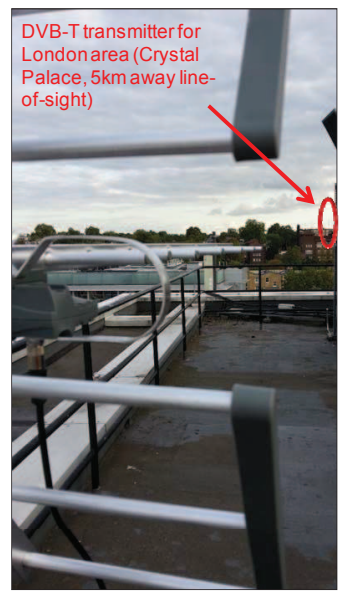

(c)
Fig. 5. Antenna configurations for DTV coexistence assessments: (a) antennas pointing in different directions, (b) antennas pointing in approximately the same direction, (c) view along the DTV antenna to the Crystal Palace London-area TV transmitter. 
Using Wavecom Wavesys DTV monitors [15], the DTV performance has been assessed by various means, including audio/visual and statistical analysis. No effect of the WSD on the DTV picture or audio has been observed. Figure 6 presents the most challenging case of a 256-QAM DTV signal being received in the adjacent channel to a transmitting WSD at maximum allowed EIRP, in the form of the constellation of the received DTV signal. Noting also that this is for the morechallenging antenna configuration in Figure 5(b), no effect of the WSD on the DTV constellation has been observed.

Regarding PMSE assessment, and referring to Figure 7, the WSD has been configured to transmit at maximum allowed power ( $31 \mathrm{dBm}$ in this case), in the adjacent channel to the PMSE, with the PMSE tuned as close as allowed to the WSD, and the WSD at $5 \mathrm{~m}$ distance and pointing directly at the PMSE receiver. The PMSE was an analogue FM wireless microphone, which would be immediately (audibly) affected by interference. The PMSE transmitter was located $6 \mathrm{~m}$ away from the PMSE receiver, highly attenuated (measured as $\sim 20$ $\mathrm{dB}$ attenuation) by placing it behind a metal cabinet.

A range of audio recordings were made over the PMSE link using audio test files, with and without the WSD transmitting. In listening tests, it has been impossible to hear any audible effect of the transmitting WSD on the PMSE link.

\section{CONCLUSION}

The Ofcom TV White Spaces (TVWS) Pilot represents an important milestone in the realisation of TVWS technology. This paper has described trials that are being undertaken in this pilot by an extensive consortium. It has also detailed some initial results that have been obtained, still at a relatively early stage in this trials. It is noted that further investigations as part of the trials have and are being done, with more results likely to be presented in further future publications.

\section{ACKNOWLEDGMENT}

This work has been supported by the "ICT-ACROPOLIS Network of Excellence", www.ict-acropolis.eu, the "Spectrum Overlay through Aggregation of Heterogeneous Dispersed Bands" project, ICT-SOLDER, www.ict-solder.eu, and partially supported by CRS-i, www.ict-crsi.eu.

\section{REFERENCES}

[1] FCC, "In the Matter of Unlicensed Operation in the TV Broadcast Bands, Additional Spectrum for Unlicensed Devices Below $900 \mathrm{MHz}$ and in the $3 \mathrm{GHz}$ Band, Second Memorandum, Opinion and Order," September 2010.

[2] FCC, "In the Matter of Unlicensed Operation in the TV Broadcast Bands, Additional Spectrum for Unlicensed Devices Below $900 \mathrm{MHz}$ and in the $3 \mathrm{GHz}$ Band, Third Memorandum, Opinion and Order," April 2012 .

[3] Ofcom, "TV white spaces - A consultation on white space device requirements," consultation, November 2012.

[4] Ofcom, "TV white spaces - approach to coexistence," consultation, September 2013 (an addendum to this from November 2013 also exists).

[5] ETSI, "White Space Devices (WSD); Wireless Access Systems operating in the $470 \mathrm{MHz}$ to $790 \mathrm{MHz}$ frequency band; Harmonized EN covering the essential requirements of article 3.2 of the R\&TTE Directive," v1.1.1, April 2014.

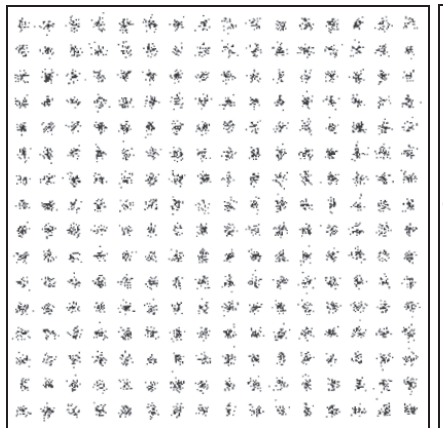

(a)

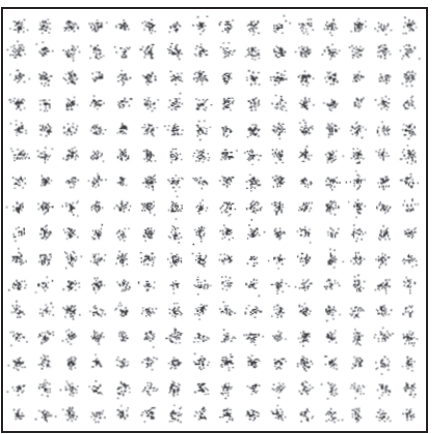

(b)
Fig. 6. 256-QAM DTV constellations observed on channel 30: (a) without the WSD transmitting, and (b) with the WSD transmitting at maximum allowed power in the adjacent channel 31.

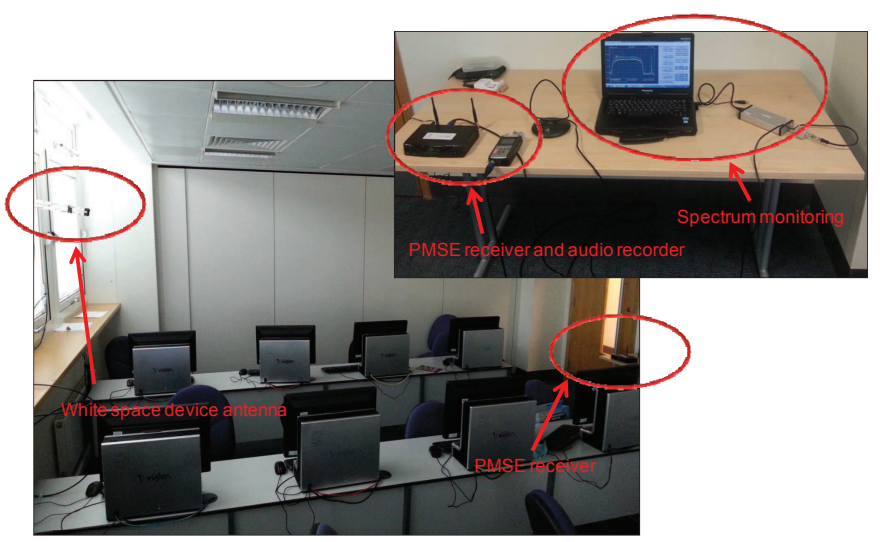

Fig. 7. Configuration of PMSE wireless mirophone coexistence experiments.

[6] Ofcom http://stakeholders.ofcom.org.uk/spectrum/tv-white-spaces/whitespaces-pilot, accessed May 2014.

[7] O. Holland, et. al., "A Series of Trials in the UK as part of the Ofcom TV White Spaces Pilot," IEEE CCS 2014, Rhine River, Germany, September 2014.

[8] NICT Press Release, "World's First TV White Space WiFi Prototype Based on IEEE 802.11af Draft Standard Developed," October 2012, accessible at http://www.nict.go.jp/en/press/2012/10/17-1.html, accessed May 2014.

[9] Takeshi Matsumura, et al., "Prototype of FDD/TDD dual mode LTE base station and terminal adaptor utilizing TV white-spaces," CROWNCOM 2014, pp. 317-322, June 2014.

[10] Eurecom

ExpressMIMO2 http://www.openairinterface.org/expressmimo2, accessed May 2014.

[11] OpenAirInterface https://twiki.eurecom.fr/twiki/bin/view/OpenAirInterface/WebHome, accessed May 2014.

[12] Carlson Wireless RuralConnect, http://www.carlsonwireless.com/ruralconnect, accessed May 2014.

[13] InterDigital White Paper, "Dynamic Spectrum Management", accessible at http://www.interdigital.com/wp-content/uploads/2012/10/InterDigitalDSM-White-Paper_Oct2012.pdf, accessed February 2015.

[14] KTS Agility White Space Radio, http://www.ktswireless.com/agilitywhite-space-radio-awr, accessed May 2014.

[15] Wavecom Wavesys DTV monitoring devices, http://www.wavecom.pt/en/noticias_detalhe.php?id_noticia=49, accessed December 2014. 